\title{
The Human Striatum is Necessary for Responding to Changes in Stimulus Relevance
}

\author{
R. Cools ${ }^{1}$, R. B. Ivry ${ }^{2}$, and M. D'Esposito ${ }^{2}$
}

\begin{abstract}
Various lines of evidence suggest that the striatum is implicated in cognitive flexibility. The neuropsychological evidence has, for the most part, been based on research with patients with Parkinson's disease, which is accompanied by chemical disruption of both the striatum and the prefrontal cortex. The present study examined this issue by testing patients with focal lesions of the striatum on a task measuring two forms of cognitive switching. Patients with striatal, but not frontal
\end{abstract}

\section{INTRODUCTION}

Cognitive flexibility is the ability to alter behavior in response to task-relevant changes in the environment. Functions required for cognitive flexibility have been associated most commonly with the prefrontal cortex (PFC) (Rougier, Noelle, Braver, Cohen, \& O'Reilly, 2005; O'Reilly, Noelle, Braver, \& Cohen, 2002; Wallis, Anderson, \& Miller, 2001; Ragozzino, Detrick, \& Kesner, 1999). In particular, the ability to flexibly control behavior by abstract rules has been linked with neuronal activity in the PFC (Freedman, Riesenhuber, Poggio, \& Miller, 2001; Wallis et al., 2001). Evidence from functional imaging and electrophysiological studies in humans further supports a role for the PFC in the flexible use of abstract rules (Boettiger \& D'Esposito, 2005; Barcelo, 2003). These studies have generally employed set-switching tasks in which the responserelevant dimension is specified by an abstract cue that either remains constant or changes across successive trials (for a review, see Monsell, 2003). Relative to control participants, patients with frontal lobe lesions, especially of the left hemisphere, exhibit significantly slower response times on switch trials compared with nonswitch trials (Mayr, Diedrichsen, Ivry, \& Keele, 2006; Aron, Monsell, Sahakian, \& Robbins, 2004; Keele \& Rafal, 2000; Mecklinger, Von Cramon, Springer, \& Matthes-Von Cramon, 1999; Rogers et al., 1998; Stablum, Leonardi, Mazzoldi, Umilta, \& Morra, 1994).

\footnotetext{
${ }^{1}$ Behavioral and Clinical Neuroscience Institute, University of Cambridge, UK, ${ }^{2}$ Helen Wills Neuroscience Institute, University of California, Berkeley
}

lobe lesions, were impaired in switching between concrete sensory stimuli. By contrast, both patient groups were unimpaired when switching between abstract task rules relative to baseline nonswitch trials. These results reveal a dissociation between two distinct forms of cognitive flexibility, providing converging evidence for a role of the striatum in flexible control functions associated with the selection of behaviorally relevant stimuli.
The striatum has also been hypothesized to play a critical role in cognitive flexibility (Cools, Clark, \& Robbins, 2004; Cools, Barker, Sahakian, \& Robbins, 2003; Ragozzino, Jih, \& Tzavos, 2002; Ravizza \& Ciranni, 2002; Ravizza \& Ivry, 2001; Cools, Barker, Sahakian, \& Robbins, 2001a, 2001b; Owen et al., 1992; Downes et al., 1989; Cools, van den Bercken, Horstink, van Spaendonck, \& Berger, 1984). Patients with mild Parkinson's disease (PD) exhibit significant impairment on the prototypical neuropsychological assay of cognitive flexibility, the Wisconsin Card Sorting Test and related paradigms as well as on taskswitching paradigms (Meiran \& Yehene, 2004; Pollux, 2004; Cools et al., 2003; Cools et al., 2001a, 2001b; Hayes, Davidson, \& Keele, 1998; van Spaendonck, Berger, Horstink, Borm, \& Cools, 1995; Lees \& Smith, 1983; Bowen, Kamienny, Burns, \& Yahr, 1975). The deficits on switching tasks evident in patients with basal ganglia (BG) degeneration resemble those seen in patients with lesions of the PFC (Owen et al., 1992; Brown \& Marsden, 1990; Taylor, Saint-Cyr, \& Lang, 1986; cf. Owen et al., 1993) and have also been attributed to an impairment in the flexible use of abstract task rules (Meiran \& Yehene, 2004; Swainson \& Robbins, 2001). Although it is possible that the ability to use abstract rules reflects the conjoint operation of a processing network that spans the frontal lobe and the striatum, there are reasons to question a functional contribution of the striatum to the flexible use of abstract rules based on the available neuropsychological evidence. First, the majority of taskswitching studies has involved patients with PD. PD is characterized not only by a marked reduction of dopamine in the striatum, but also by dopamine depletion in the PFC (Scatton, Rouquier, Javoy-Agid, \& Agid, 1982). 
Thus, the task-switching deficits observed in patients with PD may reflect frontal pathology.

Second, the tasks used to study cognitive flexibility generally require not only the ability to switch between abstract rules, but also other functions such as the ability to switch visual attention to newly relevant, concrete stimulus features (Rushworth, Passingham, \& Nobre, 2005; Rubinstein, Meyer, \& Evans, 2001). For example, we have conducted a set of studies (Cools et al., 2003; Cools et al., 2001a, 2001b) in which participants were required to rapidly switch between naming numbers and naming letters. On switch trials, the abstract task rule changed (e.g., from naming letters to numbers). However, the switch also required that the participants redirect their attention to a different stimulus feature (e.g., the number, not the letter). The impairment observed in the PD patients on this task could be due to difficulty with abstract rule-based switching, switching attention to newly relevant stimulus features, or some combination of these processes.

We address these problems in the current study, directly comparing the performance of patients with focal striatal lesions with patients with frontal lobe lesions on a task that separately assesses the ability to flexibly alter performance based on abstract rules and/or concrete stimulus features. Relatively few studies of cognitive function have involved patients with focal striatal lesions because lesions restricted to this structure are relatively rare, at least in comparison to the widespread availability of PD patients. Whereas focal lesions can, of course, result in secondary dysfunction of intact neural regions, these patients offer a more direct assay on performance changes associated with lesions restricted to the striatum.

Studies that have examined the cognitive performance of patients with focal striatal lesions have frequently relied on standard (neuropsychological) tests rather than selected tasks designed to tap specific cognitive processes of interest (e.g., Troyer, Black, Armilio, \& Moscovitch, 2004; Hochstenbach, van Spaendonck, Cools, Horstink, \& Mulder, 1998; Bhatia \& Marsden, 1994; cf. Rieger, Gauggel, \& Burmeister, 2003). Experimental investigations have generally been limited to single-case studies (Hay, Moscovitch, \& Levine, 2002; Swainson \& Robbins, 2001; Petty, Bonner, Mouratoglou, \& Silverman, 1996). Whereas some of these investigations have revealed nonspecific cognitive abnormalities, Swainson and Robbins (2001) reported a specific cognitive switching impairment in a patient with a rightsided lesion of the caudate nucleus. The patient had difficulty developing and varying tasks set in the context of competing, irrelevant visual information. The authors hypothesized that this impairment, observed across a range of tasks, reflected a problem in the use of abstract rules. However, it is also possible that the core problem related to a difficulty in switching their attention to newly relevant stimulus features.
We recently reported a functional magnetic resonance imaging (fMRI) study using a task that enabled the separate assessment of switching between concrete stimuli and switching between abstract rules (Cools et al., 2004). The results revealed a selective blood oxygenation leveldependent (BOLD) response in the striatum of healthy, college-age participants during concrete stimulus switching. The striatal response was specific to switching between stimuli and did not extend to switching between abstract task rules when this did not involve the selection of a novel stimulus. This pattern of activity was not observed in the PFC, which was more active for both stimulus- and rule-switch trials relative to nonswitch trials. In fact, the BOLD response in the PFC tended to be larger on rule- than stimulus-switch trials, although this effect was not statistically reliable. We concluded that the switching computation carried by the striatum is restricted to the transformation of concrete sensory information into motor responses, whereas the transformation of abstract task-rule representations into action does not require the striatum (and may involve the PFC).

Evidence from functional imaging data, however, is correlative in nature (Fellows et al., 2005) and does not provide evidence regarding the necessity of the $B G$ in concrete stimulus-based switching. Therefore, we set out to examine these two aspects of cognitive flexibility in patients with focal striatal lesions, using the same task as that employed in the fMRI study (Cools et al., 2004). Disambiguation of abstract rule-based switching and concrete stimulus-based switching should help clarify the role of the striatum in cognition and action. The selection of responses based on stimulus features can have direct consequences for the control of ongoing actions. Abstract rule switching, by contrast, has no direct instantiation in the sensory and/or motor domain; rather, it involves a higher order process that can be used to control (lower order) stimulus-based selection process.

The task allowed us to assess one additional aspect of striatal function: It required participants to either repeat or switch between right- and left-sided button presses. This motor switching manipulation varied independently from the stimulus- and rule-switching manipulations and therefore enabled comparison between the effects of striatal lesions on motor flexibility-classically associated with the striatum (Bhatia \& Marsden, 1994; Benecke, Rothwell, Dick, Day, \& Marsden, 1986)—with that on cognitive flexibility, which has been considerably understudied.

In order to assess whether the predicted deficit was specifically related to striatal damage (rather than to brain damage in general) we also tested a control patient group with PFC lesions. We predicted that patients with focal striatal lesions would be disproportionately impaired when switching between concrete stimulus features, relative to switching between abstract task rules. Conversely, we predicted that patients with frontal lobe lesions would be impaired on all types of switching. 


\section{METHODS}

\section{Participants}

\section{Recruitment}

The study was approved by the University of California, Berkeley Committee for the Protection of Human Subjects, and all participants gave written informed consent according to the Declaration of Helsinki.

Six patients with focal striatal lesions and six patients with lesions of the PFC were tested. All patients had suffered an ischemic event resulting in focal lesions centered on either the striatum or lateral PFC. Patients were identified from reviews of radiological charts and referrals from neurologists in the San Francisco Bay Area. For the frontal group, patients were excluded if the damage extended outside the frontal lobes. Six neurologically healthy control participants, matched for age and education to the patients, were recruited from the Berkeley community. All participants were right-handed.

Patients and control participants were excluded if they had a significant neurological or psychiatric condition not related directly to their stroke such as dementia or depression. On the basis of these criteria, five additional patients who had been referred for the study were excluded, either before or after completing the test battery: Two patients with striatal lesions had concomitant neurological conditions (cerebellar atrophy and traumatic brain injury). Two patients with a striatal lesion and one patient with a frontal lesion exhibited significant cognitive deficits consistent with dementia and were unable to understand the task instructions.

Lesion reconstructions for the patients are provided in Figure $1 \mathrm{~A}$ and $\mathrm{B}$. For the striatal group, the lesion was restricted to the left hemisphere in four patients and to the right hemisphere in two patients. By chance, the frontal lesions were also confined in four cases to the left, and in two cases to the right hemisphere. None of the frontal lesions extended into the striatum. All striatal lesions involved the putamen; the lesion of one striatal patient (BG1) extended into the caudate nucleus. There was evidence that the lesions also extended into the white matter (internal, external, and extreme capsules). Patients BG2 and BG4 may have experienced an additional stroke in the thalamic region. However, these lesions were contiguous with damage from the striatal

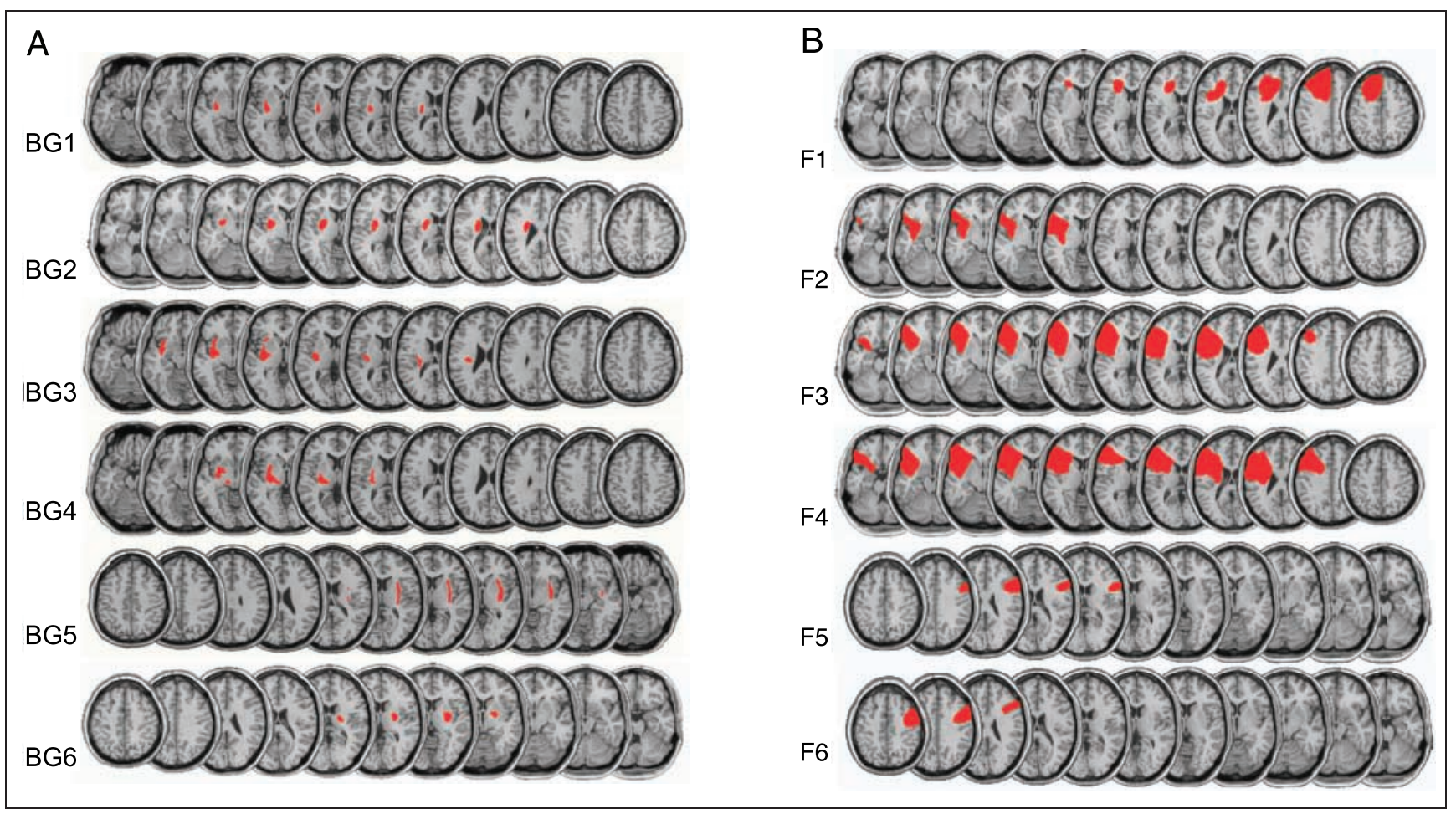

Figure 1. Extent of striatal lesions (A) and extent of frontal lobe lesions (B) as reconstructed from CT and high-resolution MRI scans. Reconstructions are presented on 11 axial slices corresponding to Talairach $z$ coordinates of $-24,-16,-8,0,8,16,24,32,40,50$, and 60 . The striatum (putamen and caudate nucleus) is visible in Sections 3-7. Figures were generated with the MRIcro software package (Rorden \& Brett, 2000; www.sph.sc.edu/comd/rorden/mricro.html) using procedures described in Brett, Leff, Rorden, and Ashburner (2001). All frontal and two striatal lesions (BG4 and BG8) were reconstructed by hand from CT scans on a normalized template by a neurologist (Dr. Robert Knight). These reconstructions were inserted into MRIcro by Clay Clayworth under the supervision of Dr. R. Knight. The remaining four striatal lesions were reconstructed directly from (i.e., drawn on top of normalized) high-resolution MRI scans by Shawn Ell and R.C., and these were checked independently for accuracy by two neurologists (Drs. Robert Knight and M.D.). None of the frontal lesions extended into the striatum. 
Table 1. Demographic and Background Details of all Participants

\begin{tabular}{lccll}
\hline & Age/Sex & $\begin{array}{c}\text { Education } \\
\text { (years) }\end{array}$ & Lesion Date & Lesion Site \\
\hline BG1 & $68 / \mathrm{m}$ & 13 & 1994 & Left putamen \\
BG2 & $81 / \mathrm{m}$ & 14 & $1974 / 1983$ & Left putamen \\
BG3 & $46 / \mathrm{m}$ & 8 & 2002 & Left putamen \\
BG4 & $54 / \mathrm{f}$ & 17 & $1992 / 2002$ & Left putamen \\
BG5 & $55 / \mathrm{m}$ & 16 & 2001 & Right putamen \\
BG6 & $65 / \mathrm{f}$ & 19 & 2000 & Right putamen \\
F1 & $60 / \mathrm{m}$ & 11 & 1998 & Left PFC \\
F2 & $46 / \mathrm{f}$ & 21 & 1997 & Left PFC \\
F3 & $46 / \mathrm{m}$ & 14 & 1996 & Left PFC \\
F4 & $42 / \mathrm{m}$ & 18 & 2002 & Left PFC \\
F5 & $70 / \mathrm{m}$ & 13 & 1990 & Right PFC \\
F6 & $54 / \mathrm{f}$ & 18 & 2002 & Right PFC \\
CS1 & $63 / \mathrm{f}$ & 14 & & \\
CS2 & $79 / \mathrm{f}$ & 18 & & \\
CS3 & $58 / \mathrm{f}$ & 17 & & \\
CS4 & $55 / \mathrm{f}$ & 14 & & \\
CS5 & $64 / \mathrm{m}$ & 16 & & \\
CS6 & $47 / \mathrm{m}$ & 12 & & \\
\hline Val & & & \\
\hline
\end{tabular}

Values represent raw scores from individual participants.

strokes. We opted to include these patients in the study. Testing was conducted at least 1 year poststroke.

The striatal patients and the control participants completed the American version of the North American Adult Reading Test (NAART; Nelson, 1982), the Beck Depression Inventory (BDI; Beck, Ward, Mendelson, Mock, \& Erbaugh, 1961) and the Mini Mental State Examination (MMSE; Folstein, Folstein, \& McHugh, 1975). One striatal patient was unavailable to complete the BDI. However, based on medical reviews, we have no reason to believe that this patient suffered clinical depression. These data, along with demographic and relevant medical information, are summarized in Tables 1 and 2. All striatal and control participants had MMSE scores in the normal range (all scores $>28$ ) and there were no significant differences between patients and age-matched control participants on the BDI or the NAART. No BDI, MMSE, or NAART data were collected for the frontal patients due to limited testing opportunities, but this group was well matched to the other two groups, both in terms of age [no group effect: $F(2,15)=$ $0.17, p=.8$ ] as well as education [no group effect: $F(2,15)=1.1, p=.4]$. The lack of background information in the frontals is unlikely to confound the results for the following reasons. First, regular evaluations by clinicians have not found evidence for depression or dementia for any of the patients. Second, the patients were matched to the controls in terms of age and education (which is a good substitute for the NAART). Third and most importantly, the results (see below) revealed a significant switching deficit only in the nondemented and nondepressed striatal patients, but not in the frontal patients. Therefore, any (unlikely) difference in terms of MMSE, BDI, or NAART scores cannot account for the observed effects. None of the striatal or frontal patients were aphasic.

\section{Experimental Task}

On each trial, participants chose one of two visual patterns. The same two stimuli were presented simultaneously, one in the left visual field and one in the right, with the location randomized. The stimuli were presented within blue or yellow stimulus windows, with the color of the two windows identical for a given trial (Figure 2). This color indicated the abstract rule to be applied in determining the response. If the windows were yellow, then the participant had to respond to the same stimulus as on the previous trial. If the windows were blue, then the participant had to respond to the pattern that had not been selected on the previous trial. Thus, some trials required that the participant switched responding between concrete stimuli (i.e., visual patterns), and some trials required that the participant switched responding between abstract rules (as indicated by the color of the boxes).

The entire stimulus display, including the patterns and cue windows, was presented until a response was made. Responses were made using the " $b$ " and " $n$ "

Table 2. Mean Demographic Data

\begin{tabular}{|c|c|c|c|c|c|c|}
\hline & Age & $\operatorname{Sex}(m: f)$ & Education (years) & $N A R T$ & $B D I$ & MMSE \\
\hline BG & $61.5(12.4)$ & $4: 2$ & $14.5(3.4)$ & $115.7(10.1)$ & $5.6(5.0)$ & $28.7(0.8)$ \\
\hline $\mathrm{PFC}$ & $53.0(10.6)$ & $4: 2$ & $15.8(3.8)$ & na & na & na \\
\hline CS & $61.0(10.8)$ & $2: 4$ & $15.0(2.1)$ & $118.7(12.4)$ & $6.8(4.9)$ & $29.7(0.8)$ \\
\hline
\end{tabular}

Values represent means (standard deviations); BDI data available for six controls and five patients (data missing from one right-sided striatal lesion patient). 
Figure 2. Sequence of five trials depicting the four trial types. The white arrows indicate the correct response. The same two patterns were presented on each trial. The yellow (here solid) stimulus windows cued participants to choose the same pattern as on the previous trial. The blue (here dotted) stimulus windows cued participants to respond to the other pattern. The feedback and fixation screens are not shown.

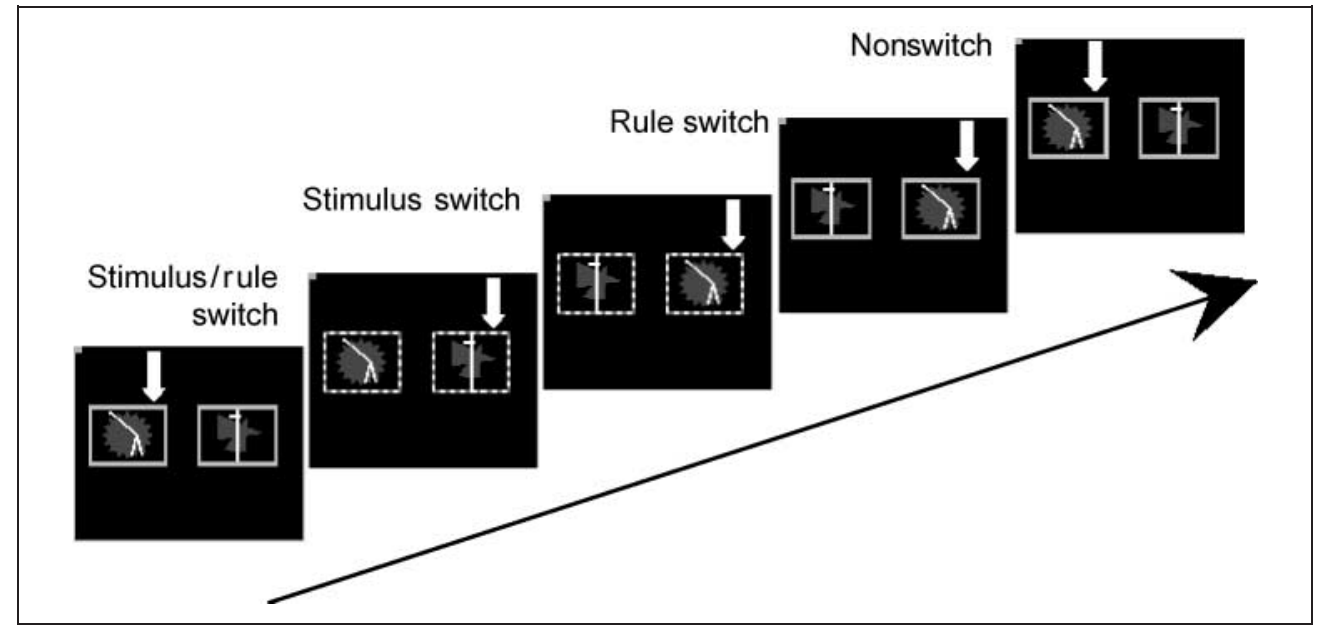

keys on a computer keyboard, with the participant indicating the right/left location of the selected pattern. Feedback, consisting of a green "smiley" face for correct responses or a red "sad" face for incorrect responses, was presented immediately after the response. The feedback face was presented for $500 \mathrm{msec}$, appearing centrally, between the two shapes. Following feedback, the stimuli were removed and the face was replaced by a fixation cross for $500 \mathrm{msec}$ prior to the presentation of the next stimulus display.

There were four trial types: (1) nonswitch trials: The rule and the target stimulus were the same as on the previous trial, that is, "yellow" trials following "yellow" trials; (2) stimulus-switch trials: The rule remained the same and the target stimulus switched, that is, "blue" trials following "blue" trials; (3) rule-switch trials: The rule switched from the previous trial and the target stimulus remained the same, that is, "yellow" trials following "blue" trials; (4) stimulus/rule-switch trials: The rule and the target stimulus switched from the previous trial, that is, "blue" trials following "yellow" trials.

Given that the left-right placement of the two stimuli was randomly determined on each trial, the design also allowed a test of response, or motor switching. For this, we compared trials on which the target stimulus appeared in the same or different locations on successive trials. This factor varied independently of rule and stimulus switching.

The rules were maintained for trials following an incorrect response. That is, if the participant made a mistake and chose the wrong target stimulus, then the correct response on the next trial was still relative to the incorrect, yet selected stimulus. We opted for this approach based on pilot testing indicating that this procedure was the least confusing for participants.

Each participant completed three blocks of 114 trials (approximately $6.3 \mathrm{~min}$ each). Stimuli were presented in a pseudorandom fixed order, so that (i) rule switching was unpredictable (the probability of a rule switch was 0.5 on each trial), (ii) the number of stimulus-repetition and stimulus-switching trials was matched within each block, and (iii) response (location) repetition was approximately matched between the four trial types.

Prior to the experimental blocks, the experimenter (R.C.) read a standard instruction paragraph and then guided performance through an initial practice block of 114 trials. Participants proceeded to the experimental blocks only if they were able to verbalize the task instructions and if they were able to complete the latter half of the practice block without guidance by the experimenter. Average error rates during the practice block for the striatal and frontal groups were $21.5 \%$ and $18.2 \%$, respectively. The data from the practice block are not included in the analysis presented here. The instructions emphasized accuracy rather than speed. Accordingly, the analyses are restricted to error rate data. Participants were allowed to use their preferred hand to respond, using the index and middle fingers of the selected hand (ipsilesional in the patients, except for two left-sided striatal patients, BG1 and BG2, who performed the task with their dominant, contralesional right hand). The experimenter left the testing room during the performance of the three experimental blocks.

\section{RESULTS}

The mean proportions of errors were calculated for each condition. For statistical purposes, an arcsine transformation was applied $(2 \times \operatorname{arcsine}[\sqrt{x}])$, to maximize the homogeneity of variances between groups. This transformation is appropriate for proportional data where the variance is a direct function of the mean (Howell, 1997). Levene's test of homogeneity of variances revealed that this transformation was successful in equalizing variance between the critical striatal and control groups (raw error rates: Levene's statistic ${ }_{1,15}=4.6, p=.06$; transformed error rates: Levene's statistic ${ }_{1,15}=0.7, p=.4$ ). Comparison of error rates from all three groups also 


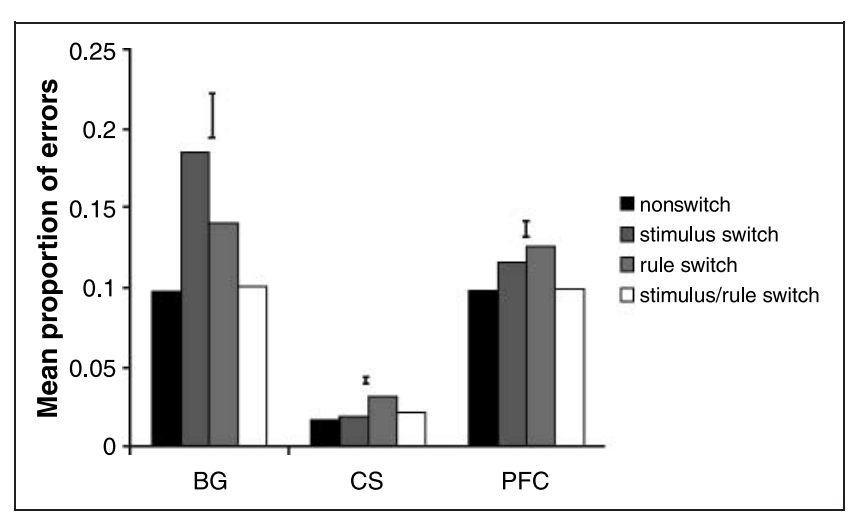

Figure 3. Mean proportion of errors as a function of group and trial type. Error bars represent standard errors of the difference between stimulus- and rule-switch trials.

revealed successful equalizing of variances (transformed error rates: Levene's statistic $2,15=1.0, p=.4$ ).

The transformed accuracy data were analyzed using a repeated measures analysis of variance (ANOVA) (SPSS 11, Chicago, IL) with one between-subjects factor (group: striatal patients, PFC patients, and controls) and one within-subjects factor: trial type (four levels: nonswitch, stimulus-switch, rule switch, stimulus/rule switch). Onetailed tests were applied for some a priori planned contrasts for which we had clear predictions with regard to the direction of the effect. Specifically, we predicted a disproportionate stimulus-switching deficit in patients with focal striatal lesions, but not in patients with frontal lesions. Greenhouse-Geisser corrections were applied when the sphericity assumption was violated (Howell, 1997).

Figure 3 presents the mean error rates for each of the four trial types. The control participants made few errors on the task, with an overall mean error rate of $2 \%$. In contrast, the error rate was considerably higher for the patients, with the striatal and frontal groups averaging $13 \%$ and $11 \%$, respectively. This main effect of group was only marginally significant, $F(2,15)=2.95$, $p_{2 \text {-tailed }}=.08$. The average error rates (for each group as well as for as each participant) were below chance (50\%), confirming that all participants understood the task instructions.

The errors were not equally distributed across the four trial types for the patients, as indicated by a significant omnibus group by trial type interaction, $F(6,45)=2.8, p_{2 \text {-tailed }}=.036$. Numerically, patients with striatal lesions made disproportionately more errors on the stimulus-switch trials relative to the rule-switch trials. In contrast, patients with PFC lesions and control participants made more errors on the rule-switch trials relative to the stimulus-switch trials.

Inspection of individuals' data, presented in Figure 4, revealed that five out of six striatal patients, but only two out of six PFC patients and zero out of six controls exhibited larger stimulus- than rule-switch costs. Interestingly, the single striatal patient who did not exhibit larger stimulus-switch costs had the smallest lesion. Furthermore, the proportion of stimulus-switch costs relative to rule-switch costs was greater for the four leftsided striatal patients (BG1-BG4) compared to the two right-sided striatal patients (BG5-BG6). This finding is consistent with our previous fMRI data, which revealed significant activity centered on the left putamen during stimulus-relative to rule switching (Cools et al., 2004). However, future studies with larger samples of right- and left-sided striatal lesions are required to evaluate laterality effects.

Decomposition of the omnibus group by trial type interaction into simple one-way ANOVAs revealed that there was a significant group effect, but only for the stimulus-
Figure 4. Difference between the proportions of errors on stimulus- and rule-switch trials for each individual participant.

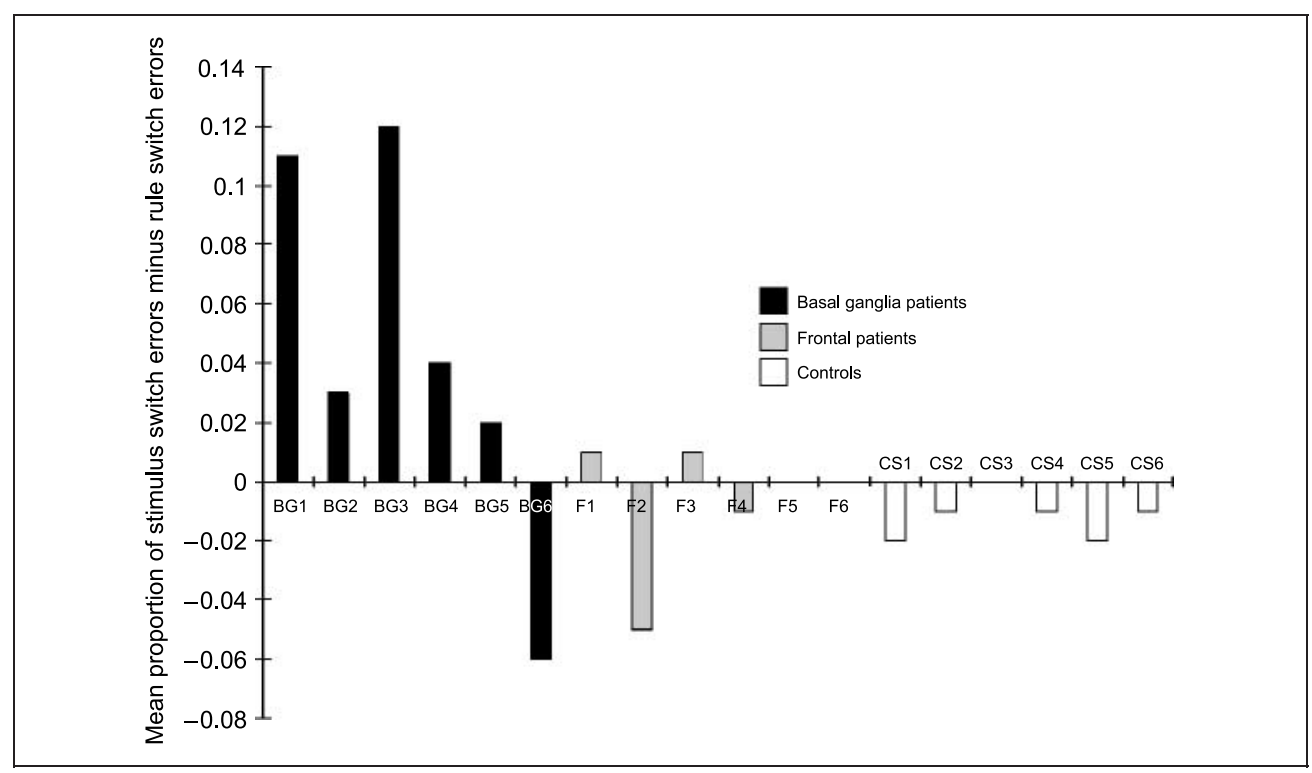


switch trials, $F(2,15)=6.1, p_{2 \text {-tailed }}=.01$, and not for the nonswitch, $F(2,15)=1.5$, rule-switch, $F(2,15)=2.6$, or stimulus/rule-switch trials, $F(2,15)=1.6$.

A first, a priori-defined contrast in which the striatal group was compared with the control group on stimulusrelative to rule-switch trials revealed a reliable interaction [group by switch-type: $F(1,10)=10.1, p_{1 \text {-tailed }}=$ .005 ], demonstrating a disproportionate switching deficit for the striatal group on stimulus-switch trials. In contrast, the interaction did not reach significance in a second, similar a priori defined comparison between the frontal lesion and control groups, $F(1,10)=2.7$. This comparison did reveal a trend toward a main effect of group, $F(1,10)=3.4, p_{2 \text {-tailed }}=.095$, and a significant main effect of switch-type, due to more rule-switch errors than object-switch errors, $F(1,10)=9.8, p_{2 \text {-tailed }}=$ .01. A third planned contrast directly compared the performance of the two patient groups. Only the interaction was reliable, $F(1,10)=4.1, p_{1 \text {-tailed }}=.034$, with the striatal patients making more errors than the frontal patients on the stimulus-switch trials only.

For completeness, we also performed analyses of simple main effects of switch-type for each group separately. Striatal patients exhibited a marginally significant increase in error rates on stimulus-switch trials relative to rule-switch trials $\left(T_{5}=1.7, p_{1 \text {-tailed }}=.07\right)$. This contrasted markedly with the significantly reduced error rate of control subjects on stimulus- relative to ruleswitch trials $\left(T_{5}=-3.4, p_{2 \text {-tailed }}=.02\right)$. The difference between error rates on stimulus- and rule-switch trials in the frontal patients was not significant $\left(T_{5}=-1.1\right)$.

Comparison of each of the patient groups with the controls on the stimulus-switch relative to the nonswitch trials confirmed a significant stimulus-switching deficit in striatal patients, $F(1,10)=9.7, p_{1 \text {-tailed }}=.006$, but not in frontal patients, $F(1,10)=1.9$. Analyses of simple main effects revealed that error rates on stimulus-switch trials were significantly greater than error rates on nonswitch trials in striatal patients $\left(T_{5}=3.6, p_{1 \text {-tailed }}=.007\right)$, but not in controls $\left(T_{5}=1.0\right)$ or in frontal patients $\left(T_{5}=\right.$ 1.0). A similar comparison of each of the patient groups with the controls on the rule-switch relative to the nonswitch trials showed that there was no rule-switching deficit in striatal patients, $F(1,10)=1.9$, or frontal patients, $F(1,10)=1.1$.

Note that the increase in error rate on stimulus-switch trials for the striatal patients was not observed on trials in which both the rule and stimulus switched. In fact, the error rate on these trials was the same as on nonswitch trials for all three groups. We will address this surprising finding in the Discussion.

To analyze effects of response repetition/alternation, we expanded the basic ANOVA to include the response variable with two levels, repetition and alternation. The main effect of response was significant, $F(1,15)=8.6$, $p_{2 \text {-tailed }}=.01$. In terms of interactions, the group by response, $F(2,15)=2.0$, and the omnibus three-way group by trial by response interaction, $F(6,45)=1.1$, were not significant, indicating that stimulus-switching impairment in BG patients was not related to response (or motor) switching. However, there was a reliable response by trial-type interaction, $F(3,45)=9.1, p_{2 \text {-tailed }}<$ .0001 . Whereas participants in all three groups were more likely to make more errors on stimulus-switch than rule-switch trials when the response was different from that on the previous trial, the reverse was observed on response repetition trials. On these trials, participants made more errors on rule-switch than stimulusswitch trials.

More importantly, the stimulus-switching impairment in striatal patients was not due to difficulty with motor or response switching: Relative to nonswitch trials, the stimulus-switching deficit was present on both response alternation, $F(2,15)=2.6, P_{1 \text {-tailed }}=.05$, as well as response repetition trials, $F(2,15)=3.0, p_{1 \text {-tailed }}=.04$. There was a trend for both patient groups to make more errors on rule-switch trials compared to controls when the response repeated relative to when the response switched. However, this effect was not reliable, as evidenced both by the lack of a significant omnibus three-way interaction (see above) as well as by a more direct test of this observation: group by trial (rule switch vs. nonswitch) by response, $F(2,15)=2.5$.

Response times (RTs) are presented in Figure 5. RTs were $\log 10$ transformed (to minimize heterogeneity of variances and maximize normality) and analyzed using a repeated measures ANOVA with group as a betweensubjects factor and trial type as a within-subjects factor. Excluded from this analysis were RTs on incorrect trials, RTs on trials following incorrect trials, RTs $>5000 \mathrm{msec}$, and RTs $<300$ msec. There was a main effect of trial type, $F(3,45)=40.7, p<.0001$, due to faster RTs on nonswitch trials than on switch trials [contrasts of each of the three switch trials with nonswitch trials: all $F(1,15)>49$, all $p<.0001]$. There was no significant

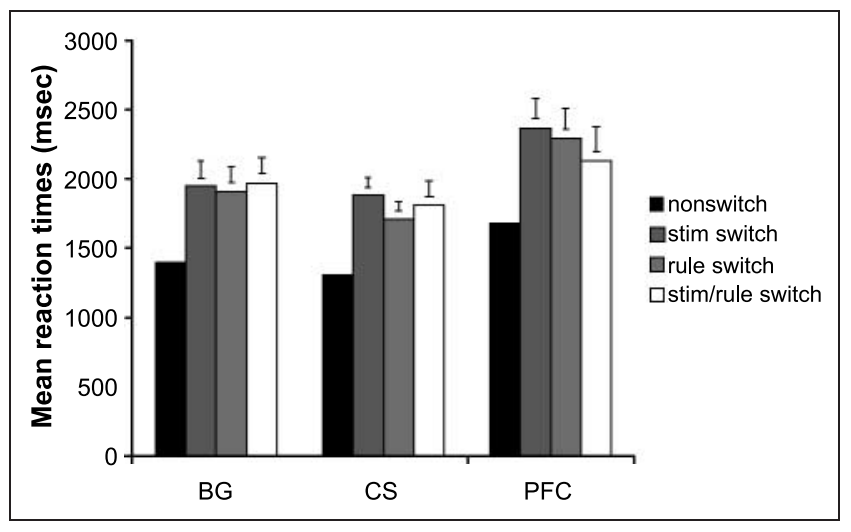

Figure 5. Mean reaction times (milliseconds) as a function of group and trial type. Error bars represent standard errors of the difference between the respective switch trials and nonswitch trials. 
effect of group, $F(2,15)=0.6$, and there was no group by trial type interaction, $F(6,45)=0.7$.

In summary, patients with striatal lesions exhibited a significant deficit on trials requiring a switch to a newly relevant, concrete stimulus. The increase in error rate on stimulus switching was significantly greater than that on rule switching. In fact, there was no evidence of an impairment in switching to a newly relevant abstract rule. Moreover, there was no evidence for a motor-switching deficit. The disproportionate stimulus-switching deficit observed in the striatal group does not appear to be a nonspecific consequence of brain damage per se, as evidenced by the relatively normal performance on switch trials relative to nonswitch trials in patients with frontal lobe lesions.

\section{DISCUSSION}

Our results reveal a cognitive switching deficit in patients with focal striatal lesions and indicate that the striatum is necessary for cognitive flexibility. The most striking feature of the current results is the observation that the striatal patients were disproportionately impaired on trials on which the response required a switch to a newly relevant stimulus pattern. The deficit in this group did not extend to trials that required a switch to a newly relevant abstract rule that did not involve the selection of a newly relevant stimulus.

The disproportionate sensitivity of stimulus-relative to rule-switching is markedly consistent with a recent observation from an fMRI study in which neurologically healthy participants were tested with the same task (Cools et al., 2004). Activation in the striatum, centered on the putamen, was elevated on stimulus-switch trials relative to rule-switch trials. Together, these fMRI and focal brain lesion studies indicate that the striatum is necessary for cognitive flexibility based on stimulus features. These data highlight the role of the striatum in the redirection of attention to newly relevant stimulus features.

The disproportionate deficit on stimulus-switch trials is unlikely to be a consequence of a general increase in task difficulty or failure to understand the task instructions. First, participants were able to verbalize the task instructions to the experimenter and performed well on a practice block. Second, error rates were well below chance. Third, the deficit was disproportionately larger for stimulus-switch trials and patients did not exhibit significant difficulty with the nonswitch, rule-switch, or stimulus/rule-switch trials. The deficit on stimulus-switch trials also cannot be accounted for by general effects of brain damage. Patients with much larger lesions in the frontal lobe did not exhibit the same performance pattern and, in fact, relative to baseline nonswitch trials, did not exhibit a deficit in either stimulus switching or rule-switching. The dissociation between the striatal and frontal groups is particularly striking given the fact that the frontal lesions were much larger than the striatal lesions.

The ability to flexibly use abstract rules has been associated with the PFC (Miller \& Cohen, 2001; Wallis et al., 2001). Based on this observation and our finding that the PFC was activated on both stimulus- and ruleswitch trials (relative to nonswitch trials) in our previous fMRI study (Cools et al., 2004), we had predicted that the frontal patients would exhibit both a stimulus- and a rule-switching deficit. The results failed to support this prediction. The increases in the error rate on both the stimulus- and the rule-switch trials compared to nonswitch trials were similar for the frontal patients and control participants. This finding is surprising in the context of our fMRI results. The divergence between the fMRI and lesion evidence may indicate that the PFC is involved in, but not necessary for the form of cognitive switching required in the present task. Alternatively, it is possible that spared switching capacities in patients with unilateral lesions might be mediated by the PFC of the intact hemisphere. By analogy, the ability to temporarily store and rehearse/maintain information is not significantly affected by unilateral frontal lesions, despite substantial brain imaging evidence that the PFC is activated during such working memory processes (D'Esposito, Cooney, Gazzaley, Gibbs, \& Postle, 2006). Memory deficits are evident only when processing within the lesioned hemisphere is taxed more directly, for example, by stimulus presentation contralateral to frontal damage (Duarte, Ranganath, \& Knight, 2005). Accordingly, by using a central stimulus presentation, our design may have underestimated prefrontal contributions.

However, there is also discrepancy between the current finding and previous studies with patients with unilateral frontal lesions that have reported significantly enhanced switch costs (Mayr et al., 2006; Aron et al., 2004; Keele \& Rafal, 2000; Rogers et al., 1998). There are a number of significant methodological differences between the current task and those employed in previous studies of cognitive flexibility. Whereas those studies have usually tested switching between stimulus dimensions (e.g., numbers and letters, color and forms), the abstract rules used here required switching between (or repetition of) stimulus exemplars. Second, previous studies have measured task-switching under time pressure. It is possible that RT measures are more sensitive than our accuracy measure (Aron et al., 2004). Thus, we recognize that the present null effect in PFC patients does not provide definitive evidence against a role for the PFC in cognitive flexibility. Although these issues await further investigation, it is important to note that the frontal lesion group provides an interesting reference point for assessing the performance of the striatal patients. In particular, the frontal group indicates that the disproportionate increase in errors on stimulusswitch trials made by the striatal patients is not simply 
due to nonspecific effects of brain damage. A similar selective increase was not found for the frontal patients.

We have emphasized that the impairment observed in the current study is related to flexible shifting between task-relevant stimuli. Our design allowed us to directly compare this form of switching with that involving the flexible use of abstract rules. It is also important to consider other functions that might be required in the current task, especially those that have been previously associated with the striatum such as reward or motor control processes. A feedback-based account cannot explain the observed dissociation between stimulusand rule-switching. Feedback (positive and negative outcomes) did not vary as a function of trial type and, thus, difficulties in using this information should have had a similar effect across trial-types. Furthermore, we did not observe a group effect in the comparison of trials involving response repetitions or response alternations. Previous studies involving patients with PD have pointed to a role for the striatum in motor switching (e.g., Hayes et al., 1998). It may be that such deficits require bilateral pathology of the basal ganglia or involvement of additional regions that are disrupted in patients with this disorder. Although null results must be considered cautiously given the small number of participants, a recent report indicates relatively intact performance of patients with unilateral striatal lesions on a range of motor tasks (Aparicio, Diedrichsen, \& Ivry, 2005).

At first sight, the stimulus-switching deficit in striatal patients may appear inconsistent with classic theory, according to which the striatum mediates the learning and memory of consistent relationships between stimuli and responses leading to habitual "priming" of responses upon stimulus presentation (White, 1997). For example, Mishkin, Malamut, and Bachevalier (1984) have suggested that the striatum subserves a slow, incremental, "less cognitive, more rigid" form of memory as opposed to the "more cognitive, flexible, and less rigid" form of memory. In keeping with these ideas, striatal lesions in animals weaken stimulus-response habits, leading to behavior being more sensitive (i.e., flexible) to changes such as reinforcer devaluation (Yin, Knowlton, \& Balleine, 2006). The present findings suggest that the striatum supports some aspects of flexible behavior. Specifically, striatum-mediated flexibility may be restricted to situations where there is change in response-relevant sensory input and does not extend to the updating of abstract rules when the same stimulus needs selected. More importantly, the current task does not require the resetting of links between stimuli and responses. What changes is the stimulus to be selected (and only indirectly its associated response). Thus, the data indicate that the striatum is required for the redirection of attention to different stimuli that elicit behavioral responses. In this sense, the role of the striatum in cognitive flexibility is not inconsistent, but rather may coexist with a role in the gradual formation of habits, or inflexible links between stimuli and responses.

One aspect of our data is quite puzzling: Although the striatal patients were impaired on stimulus-switch trials, they were not impaired on trials in which the rule switched and this change required a switch in the stimulus (i.e., stimulus/rule-switch, or yellow cue followed by blue cue). One might have expected that impairment in stimulus-based switching would also be manifest in this latter condition. This finding is especially surprising given that the stimulus/rule-switch trials were associated with a large increase in striatal activation (Cools et al., 2004). The failure to observe an impairment here suggests that the striatum is not necessary for stimulus switching in a rule-switching context. Perhaps the rule switch induced a bias to apply a switch operation in a relatively generic manner (see De Jong, Liang, \& Lauber, 1994). Thus, the cue to switch the rule may have biased the participants to also switch to the other stimulus pattern, a bias that in this case would lead to a correct response. Indeed, supplementary analysis revealed that across groups the error rate on rule-switch trials was significantly higher than that on stimulus/rule-switch trials, $F(1,15)=8.5, p_{2 \text {-tailed }}=.01$. Moreover, the error rate on the stimulus/rule-switch trials was no higher than that observed for the nonswitch baseline condition for all three groups. Further support for the idea that there was a tendency to generically apply a "switch" operation on rule-switch trials comes from the fact that participants made more errors on response repetition trials compared to response alternation trials in the ruleswitch condition only.

\section{Acknowledgments}

This work was supported by the National Institutes of Health (NIH Grants MH63901 and NS40813) and the Veterans Administration Research Service. We thank Natalie Marchant for testing some patients and age-matched control participants, and Donatella Scabini, Leslie Shupenko, and Clay Clayworth for advice and technical support. We are also grateful to Shawn Ell and Robert Knight for help with lesion reconstruction and patient recruitment. R. C. holds a Royal Society Dorothy Hodgkin research fellowship.

Reprint requests should be sent to Roshan Cools, Behavioural and Clinical Neuroscience Institute, Department of Experimental Psychology, University of Cambridge, Downing Street, Cambridge, CB2 3EB, United Kingdom, or via e-mail: roshan. cools@gmail.com.

\section{REFERENCES}

Aron, A., Monsell, S., Sahakian, B., \& Robbins, T. (2004). A componential analysis of task-switching deficits associated with lesions of left and right frontal cortex. Brain, 127, 1561-1573.

Aparicio, P., Diedrichsen, J., \& Ivry, R. (2005). Effects of focal basal ganglia lesions on timing and force control Brain and Cognition, 58, 62-74. 
Barcelo, F. (2003). The Madrid Card Sorting Test (MCST): A task switching paradigm to study executive attention with event-related potentials. Brain Research, Brain Research Protocols, 11, 27-37.

Beck, A. T., Ward, C. H., Mendelson, M., Mock, J., \& Erbaugh, J. (1961). An inventory for measuring depression. Archives of General Psychiatry, 11, 561-571.

Benecke, R., Rothwell, J. C., Dick, J. P, Day, B. L., \& Marsden, C. D. (1986). Performance of simultaneous movements in patients with Parkinson's disease. Brain, 109, 739-757.

Bhatia, K. P., \& Marsden, C. D. (1994). The behavioural and motor consequences of focal lesions of the basal ganglia in man. Brain, 117, 859-876.

Boettiger, C. A., \& D'Esposito, M. (2005). Frontal networks for learning and executing arbitrary stimulus-response associations. Journal of Neuroscience, 25, 2723-2732.

Bowen, F. P., Kamienny, R. S., Burns, M. M., \& Yahr, M. D. (1975). Parkinsonism: Effects of levodopa treatment on concept formation. Neurology, 25, 701-704.

Brett, M., Leff, A. P., Rorden, C., \& Ashburner, J. (2001). Spatial normalization of brain images with focal lesions using cost function masking. Neuroimage, 14, 486-500.

Brown, R., \& Marsden, C. (1990). Cognitive function in Parkinson's disease: From description to theory. Trends in Neurosciences, 13, 21-29.

Cools, A. R., van den Bercken, J. H. L., Horstink, M. W. I., van Spaendonck, K. P. M., \& Berger, H. J. C. (1984). Cognitive and motor shifting aptitude disorder in Parkinson's disease. Journal of Neurology, Neurosurgery and Psychiatry, 47, 443-453.

Cools, R., Barker, R. A., Sahakian, B. J., \& Robbins, T. W. (2001a). Enhanced or impaired cognitive function in Parkinson's disease as a function of dopaminergic medication and task demands. Cerebral Cortex, 11, 1136-1143.

Cools, R., Barker, R. A., Sahakian, B. J., \& Robbins, T. W. (2001b). Mechanisms of cognitive set flexibility in Parkinson's disease. Brain, 124, 2503-2512.

Cools, R., Barker, R. A., Sahakian, B. J., \& Robbins, T. W. (2003). L-Dopa medication remediates cognitive inflexibility, but increases impulsivity in patients with Parkinson's disease. Neuropsychologia, 41, 1431-1441.

Cools, R., Clark, L., \& Robbins, T. W. (2004). Differential responses in human striatum and prefrontal cortex to changes in object and rule relevance. Journal of Neuroscience, 24, 1129-1135.

De Jong, R., Liang C.-C., \& Lauber, E. (1994). Conditional and unconditional automaticity: A dual-process model of effects of spatial stimulus-response correspondence. Journal of Experimental Psychology: Human Perception and Performance, 20, 731-750.

D'Esposito, M., Cooney, J. W., Gazzaley, A., Gibbs, S. E. B., \& Postle, B. R. (2006). Is the prefrontal cortex necessary for delay task performance? Evidence from lesion and fMRI data. Journal of the International Neuropsychological Society, 12, 1-13.

Downes, J. J., Roberts, A. C., Sahakian, B. J., Evenden, J. L., Morris, R. G., \& Robbins, T. W. (1989). Impaired extra-dimensional shift performance in medicated and unmedicated Parkinson's disease: Evidence for a specific attentional dysfunction. Neuropsychologia, 27, 1329-1343.

Duarte, A., Ranganath, C., \& Knight, R. T. (2005). Effects of unilateral prefrontal lesions on familiarity, recollection, and source memory. Journal of Neuroscience, 25 , 8333-8337.

Fellows, L. K., Heberlein, A. S., Morales, D. A., Shivde, G., Waller, S., \& Wu, D. H. (2005). Method matters: An empirical study of impact in cognitive neuroscience. Journal of Cognitive Neuroscience, 17, 850-858.

Folstein, M. F., Folstein, S. E., \& McHugh, P. R. (1975). "Mini Mental State": A practical method for grading the cognitive state of patients for the clinician. Journal of Psychiatric Research, 12, 189-198.

Freedman, D., Riesenhuber, M., Poggio, T., \& Miller, E. (2001). Categorical representation of visual stimuli in the primate prefrontal cortex. Science, 291, 312-316.

Hay, J., Moscovitch, M., \& Levine, B. (2002). Dissociating habit and recollection: Evidence from Parkinson's disease, amnesia and focal lesion patients. Neuropsychologia, 40, 1324-1334.

Hayes, A. E., Davidson, M. C., \& Keele, S. W. (1998). Towards a functional analysis of the basal ganglia. Journal of Cognitive Neuroscience, 10, 178-198.

Hochstenbach, J., van Spaendonck, K. P., Cools, A. R., Horstink, M. W., \& Mulder, T. (1998). Cognitive deficits following stroke in the basal ganglia. Clinical Rehabilitation, 12, 514-520.

Howell, D. C. (1997). Statistical methods for psychology. Belmont: Wadsworth.

Keele, S., \& Rafal, R. (2000). Deficits in task set in patients with left prefrontal cortex lesions. In S. Monsell \& J. Driver (Eds.). Control of cognitive processes: Attention and performance XVIII (pp. 627-651). Cambridge: MIT Press.

Lees, A. J., \& Smith, E. (1983). Cognitive deficits in the early stages of Parkinson's disease. Brain, 106, 257-270.

Mayr, U., Diedrichsen, J., Ivry, R., \& Keele, S. W. (2006). Dissociating task-set selection from task-set inhibition in prefrontal cortex. Journal of Cognitive Neuroscience, $18,14-21$.

Mecklinger, A., Von Cramon, D. Y., Springer, A., \& Matthes-Von Cramon, G. (1999). Executive control functions in task switching: Evidence from brain injured patients. Journal of Clinical and Experimental Neuropsychology, 21, 606-619.

Meiran, N., Friedman, G., \& Yehene, E. (2004). Parkinson's disease is associated with goal setting deficits during task switching. Brain and Cognition, 54, 260-262.

Miller, E., \& Cohen, J. (2001). An integrative theory of prefrontal cortex function. Annual Review of Neuroscience, 24, 167-202.

Mishkin, M., Malamut, B., \& Bachevalier, J. (1984). Memories and habits: Two neural systems. In G. Lynch, J. L. McGaugh, \& N. M. Weinberger (Eds.). Neurobiology of learning and memory (pp. 65-77). New York: Guildford Press.

Monsell, S. (2003). Task switching. Trends in Cognitive Sciences, 7, 134-140.

Nelson, H. E. (1982). National Adult Reading Test (NART) test manual. Windsor, UK: NFER-Nelson.

O'Reilly, R., Noelle, D., Braver, T., \& Cohen, J. (2002). Prefrontal cortex and dynamic categorization tasks: Representational organization and neuromodulatory control. Cerebral Cortex, 12, 246-257.

Owen, A. M., James, M., Leigh, J. M., Summers, B. A., Marsden, C. D., Quinn, N. P., et al. (1992). Fronto-striatal cognitive deficits at different stages of Parkinson's disease. Brain, 115, 1727-1751.

Owen, A. M., Roberts, A. C., Hodges, J. R., Summers, B. A., Polkey, C. E., \& Robbins, T. W. (1993). Contrasting mechanisms of impaired attentional set-shifting in patients with frontal lobe damage or Parkinson's disease. Brain, 116, 1159-1179.

Petty, R., Bonner, D., Mouratoglou, V., \& Silverman, M. (1996). Acute frontal lobe syndrome and dyscontrol associated with bilateral caudate nucleus infarctions. British Journal of Psychiatry, 168, 237-240. 
Pollux, P. (2004). Advance preparation of set-switches in Parkinson's disease. Neuropsychologia, 42, 912-919.

Ragozzino, M., Detrick, S., \& Kesner, R. (1999). Involvement of the prelimbic-infralimbic areas of the rodent prefrontal cortex in behavioral flexibility for place and response learning. Journal of Neuroscience, 19, 4585-4594.

Ragozzino, M. E., Jih, J., \& Tzavos, A. (2002). Involvement of the dorsomedial striatum in behavioral flexibility: Role of muscarinic cholinergic receptors. Brain Research, 953, 205-214.

Ravizza, S., \& Ciranni, M. (2002). Contributions of the prefrontal cortex and basal ganglia to set shifting. Journal of Cognitive Neuroscience, 14, 472-483.

Ravizza, S., \& Ivry, R. (2001). Comparison of the basal ganglia and cerebellum in shifting attention. Journal of Cognitive Neuroscience, 13, 285-297.

Rieger, M., Gauggel, S., \& Burmeister, K. (2003). Inhibition of ongoing responses following frontal, nonfrontal and basal ganglia lesions. Neuropsychology, 17, 272-282.

Rogers, R. D., Sahakian, B. J., Hodges, J. R., Polkey, C. E., Kennard, C., \& Robbins, T. W. (1998). Dissociating executive mechanisms of task control following frontal lobe damage and Parkinson's disease. Brain, 121, 815-842.

Rorden, C., \& Brett, M. (2000). Stereotaxic display of brain lesions. Behavioural Neurology, 12, 191-200.

Rougier, N. P., Noelle, D. C., Braver, T. S., Cohen, J. D., \& O'Reilly, R. C. (2005). Prefrontal cortex and flexible cognitive control: Rules without symbols. Proceedings of the National Academy of Sciences, U.S.A., 102, 7338-7343.

Rubinstein, J. S., Meyer, D. E., \& Evans, J. E. (2001). Executive control of cognitive processes in task switching. Journal of Experimental Psychology: Human Perception and Performance, 27, 763-797.
Rushworth, M. F., Passingham, R. E., \& Nobre, A. C. (2005). Components of attentional set-switching. Experimental Psychology, 52, 83-98.

Scatton, B., Rouquier, L., Javoy-Agid, F., \& Agid, Y. (1982). Dopamine deficiency in the cerebral cortex in Parkinson disease. Neurology, 32, 1039-1040.

Stablum, F., Leonardi, G., Mazzoldi, M., Umilta, C., \& Morra, S. (1994). Attention and control deficits following closed head injury. Cortex, 30, 603-618.

Swainson, R., \& Robbins, T. W. (2001). Rule-abstraction deficits following a basal ganglia lesion. Neurocase, 7, 433-443.

Taylor, A. E., Saint-Cyr, J. A., \& Lang, A. E. (1986). Frontal lobe dysfunction in Parkinson's disease. Brain, 109, 845-883.

Troyer, A. K., Black, S. E., Armilio, M. L., \& Moscovitch, M. (2004). Cognitive and motor functioning in a patient with selective infarction of the left basal ganglia: Evidence for decreased non-routine response selection and performance. Neuropsychologia, 42, 902-911.

van Spaendonck, K. P. M., Berger, H. J. C., Horstink, M. W. I., Borm, G. F., \& Cools, A. R. (1995). Card sorting performance in Parkinson's disease: A comparison between acquisition and shifting performance. Journal of Clinical and Experimental Neuropsychology, 17, 918-925.

White, N. M. (1997). Mnemonic functions of the basal ganglia. Current Opinion in Neurobiology, 7, 164-169.

Wallis, J., Anderson, K., \& Miller, E. (2001). Single neurons in prefrontal cortex encode abstract rules. Nature, 411 , 953-956.

Yin, H. H., Knowlton, B. J., \& Balleine, B. W. (2006). Inactivation of dorsolateral striatum enhances sensitivity to changes in the action-outcome contingency in instrumental conditioning. Behavioural Brain Research, 166, 189-196. 